\title{
SARS-CoV-2: respiratory and gastrointestinal pathobiology of the corona virus illness, Covid- 19
}

\begin{abstract}
This paper summarizes the pathobiology of the recently discovered SARS-CoV-2 virus found to be the infectious agent of the Covid-19 illness. Coronaviruses represent a family of genetically related RNA dependent RNA polymerase viruses of likely zoonotic origin that are known to cause both respiratory and gastrointestinal illness in man and animals. The SARS-CoV-2 virus has been identified as the causative agent in the development of the infectious and highly contagious Covid-19 illness, from its initial discovery in a previously naïve population in December 2019 in Wuhan Province, China. Recent epidemiologic observations indicate that the infective and highly contagious nature of the virus led to the declaration of a world-wide dissemination of the virus and resulting in the classification as a pandemic by the World Health Organization (WHO) within 4 months of its original discovery. By January 2020, the virus had begun to spread to other countries, with the first documented cases in the USA in 22January 2020 and in several European nations soon thereafter. While the virus currently has no known cure, recently developed vaccines appear preventative. In addition, several therapeutic agents in concert with supportive measures have demonstrated significant promise in the development of clinical management strategies for Covid-19 illness. If proven successful, some of these therapeutic measures 26 may dramatically reduce the morbidity and mortality rates observed in the current Covid-19 pandemic.
\end{abstract}

\author{
Volume I2 Issue 3 - 202I
}

\author{
Orien L Tulp, ${ }^{1,2,3}$ Aftab R Awan,' George P \\ Einstein $^{1,2,3}$ \\ 'Department of Medicine, Colleges of Medicine and Veterinary \\ Medicine, University of Health and Humanities Virgin Islands \\ ${ }^{2}$ Department of Medicine, College of Medicine, University of \\ Science Arts and Technology, UK \\ ${ }^{3}$ Einstein Medical Institute, USA
}

Correspondence: Orien L Tulp, Department of Medicine, University of Health and Humanities College of Veterinary Medicine, USA, Email o.tulp@usat.edu

Received: May 05, 202I | Published: May 28, 2021
Objective: To review the pathobiology of the coronavirus in the respiratory and gastrointestinal tracts.

\section{Introduction}

The corona viruses represent a family of genetically related RNA viruses that are known to cause illness in bats, birds and mammals. ${ }^{1-3}$ The recent discovery of the latest member of the SARS Corona Virus family, SARS-CoV-2, currently referred to as the Covid-19 virus has rapidly spread throughout the world, affecting unsuspecting peoples of all nearly Nations. ${ }^{4}$ This highly infectious virus was declared a Pandemic by the World Health organization in March 2020, less than 4 months after its initial discovery in the Wuhan Province, China in December 2019, where the virus had already infected hundreds of patients in the weeks running up to the announcement of its official discovery by Chinese health officials. ${ }^{5-7}$ Corona viruses are known to be harbored in bats and other animals in regions of Southern Asia, ${ }^{8}$ and the Covid-19 virus is suspected to have jumped species from bats to Civets and other mammals. Civets are nocturnal cat-like animals sold as a meat delicacy in the Wuhan Province of China, the same region where the closely related SARS-CoV-2 coronavirus [2003-2004] is also believed to have originated from a potential primary reservoir in bats. ${ }^{6-8}$. The related MERS-CoV (Middle East Respiratory Syndrome (2012). also known as the 'camel flu'. MERS-CoV also causes a viral respiratory infectious illness with a high fatality rate caused by the MERS-coronavirus (MERS-CoV).$^{5-8}$. The Covid-19 Corona virus is the most recently discovered member of a family of Coronaviruses that cause human illness since their original discovery in 1960. The virus has been found to be infectious and highly contagious and causing the Covid-19 respiratory and gastrointestinal illness, often with multiorgan involvement but without a universally established treatment protocol. ${ }^{11 \mathrm{~b}}$

The gastrointestinal tract has virtually constant exposure to potentially pathogenic viruses, toxins, parasites and other microorganisms, and represents the largest component organ system of the immune system. On a molecular weight basis, the SARS-CoV-2 has the largest genome of the RNA coronaviruses yet discovered and characterized. ${ }^{9}$ Since its emergence, several potentially more infectious mutations of the coronavirus SAR-CoV-2 have been identified in the USA and other countries. ${ }^{10}$ The SARS-CoV-2 virus is among the most aggressive infectious member of the Corona Virus family yet discovered. ${ }^{11-13}$ While the full aspects of its pathobiology and propensity for infection remain incomplete or unclear at the time of this writing, much may be learned from the study of other members of the Corona virus family, and therapeutic regimens that have been found helpful in treating the symptoms of those illnesses may prove beneficial in developing therapeutic agents and treatment protocols for the current pandemic. ${ }^{12}$ Currently the SARS-CoV-2 (Covid-19) virus appears to pose the greatest health risk among the elderly and others with significant underlying health and immunologically challenged problems including myasthenia gravis where its infectivity appears more aggressive than in immunocompetent individuals. ${ }^{14}$ The contagion of the virus due to differing geographic, environmental and population density conditions is variable, especially for those who reside in the cooler climates of the planet although individuals of any locality or age including the youth may act as active carriers of the illness. ${ }^{14}$ Much may be learned from visiting other members of the Corona virus family, and which may provide valuable insight into the pathobiology and potential treatments for the newest member of this emerging family of the Corona viruses.

Currently multiple variants of the Covid 19 virus have been identified. ${ }^{9,10}$ The ' $L$ ' form (less serious, causing only mild upper respiratory (URI) illness and the more prevalent ' $S$ ' variant causing more severe URI and some gastroenterological symptoms, and which appears to account for approximately $80 \%$ of the reported Covid- 19 illnesses and the most deaths among those individuals presenting with symptoms and who were tested for the exposure to the virus in the early days of the pandemic. The overall fatality rate worldwide is still unknown likely due to untested or asymptomatic carriers, and 
significant localized undertesting, and repeat testing of previously tested individuals, especially with those presenting with minor flulike or gastrointestinal symptoms. The overall fatalities due to SARSCoV-2 appears to range between less than $1 \%$ to $4 \%$ among those tested but with significantly higher percentages having been reported among the eldest subgroup of the population..$^{10}$ It is noteworthy that the recent evolution and emergence of the SARS-CoV-2 virus has limited early detection capacities for the illness in addition to the development and production of diagnostic materials, thus clouding the actual numbers of laboratory confirmed infections. ${ }^{16}$

The usual mechanism of infection for Corona viruses including the Covid-19 to cause respiratory and gastrointestinal (GI) illness is via initial transmission by infective microdroplets by inhaling virus laden microdroplets in air, as may be discharged by a nearby carrier during an asymptomatic, pre- symptomatic or symptomatic phase of their illness ${ }^{16,17}$ In the respiratory illness, the inhaled microdroplets may impact deep in the lung or gastrointestinal tract making direct contact with and entering host cells via the angiotensin converting enzyme 2 (ACE2) receptors. ${ }^{18}$ ACE2 receptors are located on the respiratory and gastrointestinal epithelium, where they ultimately infect both Type I and Type II pneumocytes and the endothelial cell ACE2 receptors in the duodenum and small intestine..$^{17,19}$ Once the Type II pneumocytes of the respiratory epithelium and intestine are infected, the pneumocytes decrease their production of surfactant, a lipid necessary for coating the interior of the alveolar sacs and enabling them to expand and facilitate gas transfer efficiently. In the absence of surfactant, the alveolar sacs may collapse, resulting in alveolar atelectasis and acute respiratory distress syndrome (ARDS) and a significant reduction of effective lung volume and respiratory gas exchange capacity. ${ }^{19}$ The collapsed alveoli in ARDS may persist and reduce alveolar gas exchange and respiratory volume even after several years of recovery from the illness. The infective process may often occur before the unsuspecting carrier is aware that he or she may have contracted the virus and may or may not have displayed active symptoms of the illness. Reports have shown in the early days of this pandemic that the overwhelming majority $(\sim 80 \%)$ of younger individuals (under the age of 60 and without comorbidities) who have been confirmed to have an active Covid-19 illness may display milder symptoms, not unlike those of a mild cold or flu-like illness, and often recover with or without further treatment within one to two weeks' time. This includes most otherwise healthy children and younger adults, whose immune and respiratory systems may respond more quickly and robustly than older adults over the age of 60 and including those with confounding comorbidities such as chronic obstructive pulmonary disease (COPD), diabetes mellitus (DM) and hypertension. ${ }^{7}$ Older patients aged 80 or higher and those with musculoskeletal disorders including myasthenia gravis have exhibited the highest fatality rates to date, often associated with diminished immunocompetency and other confounding morbidities than in younger populations. ${ }^{7,15}$ Due to the mild symptoms displayed by many infected patients who may not have been tested, the actual rates of infection are largely unknown due to likely undertesting and underreporting to public health agencies. ${ }^{16}$

Many Covid-19 patients may present with gastrointestinal (GI) symptoms including diarrhea, vomiting and loss of appetite, with or without accompanying respiratory symptoms ${ }^{7,10,14,18}$ A second likely mechanism of infection linked to both respiratory and gastrointestinal infection is by hand to face contact with a recently discharged microdroplet from a nearby contaminated surface or carrier individual, resulting in GI illness. ${ }^{7,17,20}$ The individual may also ingest contaminated particles from respiratory drainage secretions, thereby forming an efficient conduit to the GI tract. The physicochemical environment of the GI tract is variable from person to persons, particularly with the very young and with aging, and which may attenuate the impact on the GI tract due at least in part to atypical or decreased gastric acidity in those age groups. ${ }^{21}$ The capacity to infect the GI tract also likely depends on the viral load ingested, the relative acidity of the stomach, and the enzymatic digestive capacity of the duodenum, including the exocrine pancreatic and small intestinal enzymes. ${ }^{22}$ The gastrointestinal tract secretions may interact with both the glycoprotein spikes and the coronal lipoid membrane. The GI-induced biochemical alterations of the outer surfaces of the viral particles and/or the lipoid membrane may occur, either of which may decrease virus-to-cell receptor attachment, the viral load, and the proposed relative infectivity potential of the viral particle once the infective components are biochemically denatured.

Infective Corona viruses are so named because of the crown shaped structure of their envelope, which becomes covered with the characteristic multiple glycoprotein viral knobs over the outer surface of their envelope, and which are responsible for key elements of the infective process via direct contact with the ACE2 receptors. ${ }^{3}$ The physicochemical composition of the envelope is predominantly lipoid in nature, thus enabling scientists to easily disrupt the envelope with commonly available lipid solvents containing either, chloroform or alcohols (especially ethanol or isopropyl alcohol) all of which can denature and disrupt the continuity of the lipoid envelope and may rapidly render the virus avirulent, whether it be as an airborne microdroplet or surface-connected viral particle. Mild heating of aerosolized microdroplets, the predominant route of infection of Covid-19 also decreases the infective potential of the viral particles, owing to the disruptive thermal effects on the lipoid composition, and which also appear to facilitate the rendering of the previously infective particles less virulent as an intact lipoid envelope with the glycoprotein viral knobs are deemed essential elements for the infectious contagion of the viral particles. ${ }^{22}$ All enveloped viruses are sensitive to ether, chloroform, or any lipid solvent, proteases, lipases and to heat and relative humidity. ${ }^{14-17}$ There are several different types of corona viruses which affect different systems in our body and also a variety of different mammalian animal species. The Corona virus which causes GIT illnesses are typically resistant to stomach acid and the alkaline $\mathrm{pH}$ in the intestine and result in virally caused enteritis or gastroenteritis. Similarly, the environmental airborne survival of Corona viruses which causes colds and other respiratory illness are often inversely affected by cool dry air in the winter or cooler summer months, which extends their survival once outside the body. In contrast, greater heat and high humidity decrease the environmental survival of the virus. ${ }^{16}$ Since the lung interior surfaces are normally 2 to 2.5 degrees cooler than the core temperatures, this may also appear to enhance the viral survival and infectivity once a host is infected. In contrast, the higher ambient temperatures and greater humidity during the normal summer months especially in tropical climates may compromise pre-infection viral survival to some Corona viruses, which may contribute to causes of respiratory illness to diminish, with a predicted decrease in the epidemic proportions of the virus. The role of environmental ultraviolent radiation exposure on viral viability common to warmer tropical environments on microdroplet transmission has not been fully determined but may also contribute to the potential for virus transmission. Crowded environments including close person to person contact that impede social distancing however may complicate environmental factors impacting on viral transmission even in the warmest climates

Corona and other viruses also appear to have different strengths of their envelopes. ${ }^{17}$ Without an envelope or with a severely damaged 
envelope the virus typically becomes avirulent. It may be wrong to address that respiratory corona virus agents are all the same as in case of the MERS coronavirus. The MERS corona virus is slightly more heat resistant as compared to the cold resistant corona virus strains, but it still is sensitive to extreme heat and that is proposed among the factors why the MERS-CoV may have died out in in hot summer months of the Middle Eastern summer heat. Both the glycoprotein and lipid composition of knobs on the envelope are necessary for virus attachment and penetration to the susceptible epithelial and gastrointestinal cells.

In a recent study, gastrointestinal symptoms were reported in nearly half $(48.5 \%)$ of 204 confirmed cases of Covid- $19^{7}$. Owing to the physicochemical nature of the lipoid envelope, the infective viral particles may be only minimally affected by exposure to mild acids or alkalies, such as may be encountered in the gastrointestinal tract. In contrast, stronger alkalies or acids are likely to exert a denaturing effect on the lipoid-glycoprotein envelope complex as well as the binding affinity of the $\mathrm{pH}$ sensitive nature of the glycoprotein spike proteins. While the stomach acids may reflect a $\mathrm{pH}$ as low as 2.0 in most otherwise healthy adults, the center of an ingested food bolus may remain enzymatically active at a slightly alkaline $\mathrm{pH}$ for an hour or more after ingestion of infected food components, and thus potentially harbor an active and viable ingested corona virus particle and shield it from immediate digestive denaturation. Should the virus survive the upper gastrointestinal tract, it may precipitate gastrointestinal symptoms including loss of appetite, emesis, diarrhea and GI neurologic inolvement. ${ }^{22}$ However, the glycoproteins associated with the viral knobs of Covid-19 would likely be potentially denatured by the stronger digestive actions of the upper GI tract, ${ }^{21}$ rendering the particles less virulent or avirulent. Protease inhibitors as may be found in a number of experimental agents and natural products including the commonly used spice oregano, may also render the particles avirulent, and may attenuate the course of development of the respiratory and gastrointestinal illness. Because the very young and the very old typically produce less stomach acids including hydrochloric acid $(\mathrm{HCl})$, this may render them more susceptible as active carriers. In the older more aged populations, whose immunocompetence may also be in decline, often suffer more severe illnesses and comorbidities due to Covid-19 than younger or middle aged patients. ${ }^{7,20}$

Historically pandemics have appeared somewhere on the planet about three times a century. ${ }^{1-3}$ Viruses and microorganisms are known to mutate from time to time, some as often as annually and with resulting variations in their infectivity rates in the new strains which may contribute to their potential to develop into an endemic or pandemic in a naïve population before the establishment of herd immunity. ${ }^{8,26}$ Unlike the common segmented flu virus one may see a new strain of the flu-causing virus emerge almost every year due to a point mutation or antigenic drift in the viral genome whereby a new virus emerges due to the antigenic shift. This characteristic has been observed to create a new virus almost every 15 to 25 years and resulted in the pandemic-like in Spanish flu of 1918 and other flu pandemics when there was not yet an established reservoir of immunity to the new viral strain in human populations. Genetic recombination could also occur between the genomes of related viruses. The most recent genetic recombination was in a transmissible gastroenteritis virus of swine (TGS) which was endemic in Denmark for centuries. However, in 1996 the TGS virus mutated, and a respiratory variant of the transmissible gastroenteritis virus was isolated. ${ }^{25}$ This new deletion mutant lost the tropism for the swine gastrointestinal track but gained the tropism for the respiratory system and then began spreading by air microdroplets rather than by the oral route or by contamination of foliage normally consumed by the swine. ${ }^{20-25}$ It is inciteful to review the mutations of the past to gain insight regarding potential successful treatment regimens for newly mutated viruses as it is likely probable that a considerable proportion of the antigenic epitopes and metabolic characteristics of the ancestral virus may be preserved in the newly emerging viruses. In the case of SARS-CoV-2, between $70 \%$ and $88 \%$ of the antigenic epitopes located on the spike (S) glycoproteins were found to be preserved forward from the SARS-CoV of 2004 to the current Covid-19 strain and suggesting a possible overlap in effective therapeutic measures one might find in the new viral strain. ${ }^{26}$ It is noteworthy that the initial spike protein ACE2 priming transmembrane protease of SARS-CoV, MERS-CoV and SARS-CoV-2 all impinge on the same ACE2 receptor of target tissues and protease inhibitors have been suggested as a possible mechanism to treat early stages of the infection. ${ }^{27}$ Indeed, the drugs used in the treatment of SARSCoV (2003-2004) have recently been observed to be at least partially effective in reducing the viral load and duration of illness of SARSCoV-2 of 2019 as they had for earlier outbreaks of related coronavirus mediated illness, thereby sparking a need for additional carefully designed clinical trials necessary to confirm the initial results. ${ }^{28}$ While there currently is no known cure for the Covid-19 illness per se a number of pharmaceutical and biomedical agents have been used to treat the illness with at least some success. A number of the older pharmaceutical agents have also been investigated for their potential to treat the SARS-CoV-2 illness since the onset of the pandemic.

The prodrug Remdesivir was used via the 'compassionate use' privilege to treat the recent West African Ebola virus epidemic of 2013-2016. ${ }^{26-30}$ Remdesivir is metabolized by the host into its active form, GS- 44152426-30. The GS-441524 metabolite is an adenosine nucleotide analog that interferes with the biochemical actions of viral RNA polymerase and would appear to evade the proofreading step by a viral exoribonuclease (ExoN) enzyme. In so doing, ExoN. may induce a diminution in net viral RNA production by the infected host cells. It remains unknown whether GS-441524 causes mutations in the RNA product or if it terminates the biosynthesis of the viral RNA chains. ${ }^{4}$ However, since it has been reported that the RNA-dependent RNA polymerase of the ebolavirus was inhibited for the most part by delayed chain termination it is likely that the same or similar mechanism is enacted in Coronavirus infected cells. ${ }^{26-30}$ Laboratory tests suggest Remdesivir is likely effective against a wide range of viruses, including SARS- $\mathrm{CoV}$ and MERS-CoV both of which have many biochemical similarities with the SARS-CoV-2 virus and may shorten the duration of hospital stay by up one third in patients administered Remdesivir. ${ }^{27-31}$ Numerous other agents with antiinflammatory actions have also been reviewed with variable results, including ivermectin and hydroxychloroquine, both of which are readily available and in approved use in moderate dosages for other illnesses worldwide for many years. The natural phenolic resveratrol, capable of stimulating production of fetal hemoglobin where it enhances the capacity for myoglobin oxygenation, has also been reported to inhibit the replication of the coronavirus in vitro and thus may have therapeutic potential here. ${ }^{32,33}$ The likely key to success in treating SARS-CoV-2 and other infectious illnesses regardless of the agent or agents considered is early treatment, when the immune system is likely to be less compromised and before later term complications and comorbidities have developed to a point where such treatments are likely to be futile.

The application of UV light and/or UV blood treatments have not been explored for SARS-CoV2, but the experience with the Ebola virus, although genetically distinct from the Covid-19 virus, found the Ebola virus to be sensitive to environmental UV light exposure in 
clinical environments where it can be used to disinfect water and air in the wavelength $200-100 \mathrm{~nm}$. (UVC).$^{34}$ This may suggest that room environmental air exposure to UV light may also facilitate the demise of the Covid-19 viral laden microdroplets in a clinical environment. ${ }^{16}$ Both the UV light and the UV blood irradiation have been found useful in treating and controlling the infectivity of other infectious viruses and microorganisms, where the production of cytokines was shown to have increased in patients following the UV blood treatment. ${ }^{35-37}$ It is noted that individuals being exposed to UV light are advised to wear protective glasses to minimize risks of retinal injury during exposure, while no such vision precaution is deemed necessary for the UV blood treatment since there is normally no direct visual exposure to UV irradiation during such treatment protocols..$^{35-37}$

\section{Results and conclusions}

The review summarizes the pathobiology of Covid-19 in a naïve global human population, and subsequent passage to many countries, including the United States, Japan, Australia, France and others, all which have modern and up to date health care systems, and all within only weeks of its initial discovery. This resulted in the declaration of a global pandemic by the WHO within less than 4 months of its original discovery as reported to the WHO in late December 2019. Epidemiologic indicators indicate that SARS-CoV-2 virus evolved from the coronavirus family, capable of causing respiratory and gastrointestinal illness in man and animals. One theory suggests that the infective zoonotic virus may have been passed from bats to mammalian species such as civets, thought to have been used for human meat consumption as a delicacy in the region, and likely resulting in the passing of infective microdroplets via the respiratory or gastrointestinal routes to a naïve human population. Viral transmission seems highly likely to be analogous to that of other similar viruses, including both airborne microdroplet inhalation of infective particles, in addition to virus laden surface contaminants derived from the discard of infected microdroplets and taken into the naïve host by inhalation, ingestion or both venues. Environmental conditions conducive with the most effective transmission include cool dry air typical of Northern climates and high population density, and with lesser effectiveness of transmission following social distancing and in humid tropical environments. The infective potential likely decreases and the distance of the social distancing increases due to dilution of the viral load of the microdroplets and contaminated surfaces. The infective microdroplets if left undisturbed in a warm humid environment greater than $90^{\circ} \mathrm{F}$ and $80 \%$ relative humidity may also render the virus avirulent within a few hours of existence in an ambient tropical environment. ${ }^{15,16}$ Regardless of the mode of entry to a naïve host, contact with the SARS-CoV-2 virus often results in the development of Covid-19 illness after an incubation period of approximately 5 to 14 days post exposure, and affects primarily the respiratory, gastrointestinal or both systems during the course of its pathophysiologic development. The illness may spread at an alarming rate owing to the naivety and absence of herd immunity of the global populations, having never been exposed to the current viral strain, and the resultant lack of an effective host immune response or effective antiviral agents to treat the viral infection. The aggressiveness of the infection is particularly concerning among the elders and immunocompromised individuals of a community, who often appear to be unable to generate an effective immune response in a timely manner.

While the mortality rates are lower in the overall population, ranging between 1 and $4 \%$ or less in younger members but they appear somewhat greater in the elder population of a community who often may have clinically significant comorbidities and underlying conditions. Especially susceptible common conditions found to be at risk include hypertension, cardiac, asthma, diabetes, chemotherapy and other immunocompromised status, most of which conditions although more common in elders, may be present at virtually any age. Thus, the true incidence of the illness and its associated mortality rates are yet to be confirmed due to the newness of the virus and an as yet incomplete data base. The combined contributions of under reporting among those with minor symptoms plus an inability to test all suspected or possible cases due to the nonavailability of reliable testing procedures, test centers and laboratory resources during the early days and weeks of the pandemic are also factors. What is known is that the greater the age with underlying conditions the higher the resultant mortality rate has been noted. This is important on the community level, so that families affected with the virus may attend to the best care available for the loved ones of their families. Effective therapeutic protocols and pharmacotherapeutic agents are actively under development, and currently revolve around several readily available agents currently in production. These drugs may be administered under a 'compassionate off-label use' privilege now, given alone or in combination, along with secondary drugs and regimens to minimize renal and liver function, and broad-spectrum antimicrobials to ward against the potential development of secondary infections during treatment in susceptible individuals for an illness otherwise not known to have an established curative treatment or other form of cure. The rapidity of the development of several effective vaccines during the first year of the pandemic was historic, and by the end of 2021 will likely have been administered to a majority of the global population and bring closure to the pandemic.

\section{Discussion}

The emergence of the novel corona virus SARS-CoV-2 (Covid-19), first reported in December 2019, presents a serious threat to the global community who at this time in history remains mostly naïve to the virus, having never previously been exposed. Although in early reports the elderly and those with other comorbidities have shown to pose a greater risk of mortality once infected, patients of all ages throughout the lifespan including children have now been shown to have been infected with the virus and many have succumbed to the illness. Patients throughout the lifespan may become infected and sometimes remain symptomatic with mild symptoms or no symptoms at all. In an unknown number of occurrences, the viruses may be transmitted while individuals are asymptomatic as may also well be the case in the early days of the infection. Environmental factors including a crowded population density have been observed to play a major role in its transmission, including factors of climate and an unawareness of the emerging viral threat. The infection is highly contagious and is believed to be normally spread by viral laden microdroplet inhalation or ingestion or both venues from an infected host. Once infected, the new host may experience respiratory or gastrointestinal symptoms, or both within a week or two of the exposure. Symptoms of the closely related SARS-CoV have produced similar symptoms in a variety of mammalian species that are commonly part of the food chain in most Nations and cultures, and in some cases may even have infected a family pet that may now become a carrier. The SARS-CoV-2 has been found in bats and civets, a nocturnal cat like mammal indigenous in Asia, where it is sometimes consumed as a source of meat delicacy. Currently there is no known or verified cure by double blinded investigation. The duration of any immunity that may be conferred after recovery from the virus remains unknown, but antibodies recovered from the plasma of previously infected patients have been reported to attenuate the infection in seriously ill patients. Several emerging vaccines have now been developed and granted emergency 
use by multiple governments. The first phase I vaccine clinical trials were announced in mid-March 2020, less than 4 months after the first reported cases in China (December 2019) or the USA (January 2020). The highly contagious nature of the Covid-19 illness was likely to enable it to trigger a significant spike in reported infections by March 2020 due to the previously unrestricted movements of infected individuals including both domestic and International travel in addition to unrestricted community exposure. In addition, some individuals of a community may have mistaken the early symptoms for a seasonal cold or seasonal flulike illness, and unaware that the individuals surrounding them in their close proximity were being placed at risk for a number of hours after the release of the infected microdroplets or via the fecal-oral route as shed viral particles can also be recovered from fecal matter.

Air travel regardless of duration is particularly at risk, as the low humidity as is experienced at high elevations attained during many flights has been shown to prolong the extracorporeal viral survival on infected air space and the impacted surfaces surrounding the infected individual. This risk may persist often for many hours at a time, where many innocent passersby may become infected. Since the 'dose makes the poison' dogma in toxicologic environments, the longer the flight, the greater the risk of contracting the infection via the multiple possible transmission venues. Within a week of the exposure, the innocently infected individuals may begin to spread the virus to other unsuspecting individuals also naïve to the virus. Individuals of all ages are assumed to be capable of viral transport and continued spread of the illness causing virus. The development of antiviral agents and the potential effects of contributory anti-inflammatory agents remains an active area of pharmaceutical research, with a limited number of potential agents in an FDA approved 'compassionate' off label use in severely infected patients. Of interest, while hydroxychloroquine and ivermectin, older well-established antimalarial agents with anti-inflammatory actions and an acceptable safety record spanning over 50 years emerged early in the pandemic as a readily acceptable treatment for Covid-19, given as monotherapy or in combination with other pharmaceuticals, but convincing studies supporting their best use remain unclear. In addition, a leading antiviral compound Remdesivir acts as a prodrug before conversion to an active antiviral form, where it prevents or impedes the continued replication of the virus in vivo. The virus, left unchecked infects not only the respiratory lining, including the pneumocytes, responsible to produce surfactant, necessary for alveolar expansion and gas exchange, but can also infect the gastrointestinal tract, where it may be confused with other gastrointestinal illnesses including diarrhea and GI obstruction, due to the similarity of symptoms. ${ }^{39}$ As the respiratory infection progresses, the release of surfactant becomes decreased to the point where it can precipitate acute respiratory distress syndrome (ARDS), and which if left untreated via artificial ventilation and other supportive therapeutic measures, may result in the death of the patient. Digestive symptoms may largely affect the process of COVID-19 disease because the virus enters target cells through the ACE2 receptor, which is highly expressed in cells of the GI tract. Once infected, GI epithelial cells result in cytokine and chemokine release, instigating acute intestinal inflammation characterized by infiltration of neutrophils, macrophages and $\mathrm{T}$ cells. Patients infected with the SARS-CoV-2 virus have accompanied diarrhea which may indirectly support this point. Antiinflammatory agents may attenuate the symptoms via quenching of free radicals or other markers of inflammation. Among patients who have recovered from Covid-19 and Covid- mediated ARDS, respiratory and gastrointestinal deficits may persist for weeks or months following recovery. In the case of Covid-linked ARDS the deficits have been predicted to persist for up to 5 years post viral recovery. Thus, due to the serious and often life-threatening illness that SARS-CoV-2 may present to the most vulnerable segments of the population, and the highly contagious nature of its spread, dissemination and infectivity of naïve individuals, the pressure for development of effective antiviral agents, vaccines and other effective measures to contain the virus and its myriad of developing mutations are urgent and cannot and must not be neglected. ${ }^{40-47}$

\section{Acknowledgments}

This study was supported by Institutional Resources of the University of Health and Humanities, Virgin Islands, the University of Science Arts and Technology, Montserrat, and the Einstein Medical Institute, USA. Dr Aftab R. Awan is a Life Member of Darwin College the University of Cambridge, Cambridge, UK.

\section{Conflicts of interest}

All authors declared there are no conflicts of interest.

\section{Funding}

None.

\section{References}

1. Committee on Taxonomy of Viruses (ICTV). Virus Taxonomy: 2018b Release. UK: International Committee on Taxonomy of Viruses (ICTV); 2019 .

2. International Committee on Taxonomy of Viruses "2017.012-015S”(xlsx). UK: International Committee on Taxonomy of Viruses (ICTV); 2019.

3. Murphy F, Gibbs A, Paul J, et a.. Veterinary Virology. $3^{\text {rd }}$ end. (Elsevier) pp 495-508.

4. World Health Organization. Coronavirus disease 2019 (COVID-19): situation report 37. February 25, 2020. Geneva: World Health Organization. 2020.

5. Zhu N, Zhang D, Wang W, et al. China Novel Coronavirus Investigating and Research Team. A novel coronavirus from patients with pneumonia in China, 2019. NEJM. 2020;382(8):727-733.

6. World Health Organization. "WHO Statement Regarding Cluster of Pneumonia Cases in Wuhan, China. Geneva: World Health Organization. 2020 .

7. Chen N, Zhou M, Dong X, et al. Epidemiological and clinical characteristics of 99 cases of 2019 novel coronavirus pneumonia in Wuhan, China: a descriptive study. Lancet. 2020;395(10223):507-513.

8. Cui J, Li F, Shi ZL. Origin and evolution of pathogenic coronaviruses. Nat Rev Microbiol. 2019;17(3):181-192.

9. Sexton NR, Smith EC, Blanc H, et al. Homology-Based Identification of a Mutation in the Coronavirus RNA-Dependent RNA Polymerase That Confers Resistance to Multiple Mutagens. J Virol. 2016:90(16):74157428 .

10. Tulp OL, Odeyale RF, Einstein GP, et al. Will the recent emergence of coronavirus mutations precipitate an increase in the incidence of stress disorders among health care professionals? MOJ Public Health. 2021;10(1):31-33.

11. World Health Organization. Laboratory testing of human suspected cases of novel coronavirus (nCoV) infection. Interim guidance. Geneva: World Health Organization; 2020.

12. DiRuzzo J, Kerna NA. Coronavirus Now: A Timely and Practical Summary of the Current Epidemiological Information, Clinical Presentation, and Prevention and Treatment of 2019-nCoV (2019 Novel Coronavirus). EC Emergency Medicine and Critical Care. 2020;2020:25-31. 
13. Centers for Disease Control and Prevention. Novel Coronavirus 2019 , Wuhan, China. USA: Centers for Disease Control and Prevention. (CDC). 2020 .

14. https://www.who.int/docs/default-source/coronaviruse/situationreports/20200121-sitrep-1-2019-ncov.pdf

15. Wiersinga WJ, Rhodes A, Cheng AC, et al. Pathophysiology, Transmission, Diagnosis, and Treatment of Coronavirus Disease 2019 (COVID-19): A Review. JAMA. 2020;324(8):782-793.

16. Transmission of Novel Coronavirus (2019-nCoV)| CDC. USA: Centers for Disease Control and Prevention. 2020.

17. World Health Organization. Laboratory testing of human suspected cases of novel coronavirus (nCoV) infection. Geneva; World Health Organization; 2020.

18. van Doremalen A, Bushmaker T, Morris DH, et al. Aerosol and surface stability of $\mathrm{HCoV}-19$ (SARS-CoV-2) compared to SARS- CoV-1. N Engl J Med. 2020;382(16):1564-1567.

19. Awan A, Tulp OL, Field H, et al. Immunopathology in Lungs after Intranasal Challenge with Live Virus in an EHV-1 Murine Model: Lessons Learned from Unexpected Findings. The FASEB Journal. 2020;34(1):1

20. Zhang J, Garrett S, Sun J, et al. Gastrointestinal symptoms, pathophysiology, and treatment in COVID-19. Genes Dis. 2020.10.1016/j. gendis.2020.08.013.

21. Wu C, Chen X, Cai Y. et al. Risk Factors Associated with Acute Respiratory Distress Syndrome and Death in Patients with Coronavirus Disease 2019 Pneumonia in Wuhan, China. JAMA Intern Med. 2020;180(7):934-943.

22. Pochapin MJ. American College of Gastroenterology. Podcast. 2020.

23. Coates E. Study finds unexpected coronavirus symptoms that could be first sign of infection: Digestive Issues. 2020.

24. Bende, DA. Introduction to Nutrition and Metabolism. In: Nutrition and Metabolism. USA: CRC Press; 2014. Pp. 105-108.

25. Awan AR. Personal communication. UK: URI Infectivity of student spouses in Cambridge; 2020.

26. Awan A, Tulp OL, Field H. Characterization of Viraemic Cells in a Murine Model of EHV-1 Infection. The FASEB Journal. 2020;34(S1):1-1.

27. Gao K, Nguyen DD, Wang R, et al. Machine intelligence design of 2019nCoV drugs. bioRxiv. 2020;2020.01.30.927889.

28. Murphy F, Gibbs E, Paul J, et al. Veterinary Virology. $3^{\text {rd }}$ edn. USA: Academic Press; 1999. pp 495-508.

29. Grifoni A, Sidney J, Zhang Y, et al. A Sequence Homology and Bioinformatic Approach Can Predict Candidate Targets for Immune Responses to SARS-CoV-2. Cell Host Microbe. 2020;27(4):671-680.e2.

30. Preidt R. Experimental Drug Shows Promise Against Dangerous Viruses: Medicine worked in lab tests against germs that cause SARS and MERS infections. Health Day. 2017.

31. Colson P, Rolain JC, Raoult D, et al. Chloroquine for the 2019 novel coronavirus SARS-CoV-2. Int J Antimicrob Agents. 2020;55(3):105923.
32. Sheahan TP, Sims AC, Graham RL, et al. Broad- spectrum antiviral GS5734 inhibits both epidemic and zoonotic coronaviruses. Sci Transl Med. 2017;9(396):eaal3653.

33. Wang M, Cao R, Zhang L, et al. Remdesivir and chloroquine effectively inhibit the recently emerged novel coronavirus (2019-nCoV) in vitro. Cell Research. 2020;30(3):269-271.

34. Brunk D. Remdesivir under Study as Treatment for Novel Coronavirus. Medscape. 2020.

35. Pasquereau S, Nehme Z, Ahmad, HaidaA, et al. Resveratrol Inhibits $\mathrm{HCoV}-229 \mathrm{E}$ and SARS-CoV-2 Coronavirus Replication In Vitro. Viruses. 2021;13(2):354.

36. Nwokorie U, Einstein GP, Tulp, OL. Use of trans-3'5'4-trihydroxystilbene (Resveratrol) as an adjunct in the treatment of Sickle Cell Disease. FASEB J. 2021;460(35):S1

37. Goodman and Gilman's The Pharmacological Basic of Therapeutics. 12th edn. USA: McGraw-Hill; 2011. pp. 1404-1462.

38. The Slovakia Republic's Minister of Health has formerly registered Ivermectin as an approved prophylaxis and treatment for SARS-CoV-2, the virus behind COVID-19. In breaking news, the authorization occurred yesterday as doctors received the news that they could proceed with formally authorized prescriptions both in hospitals and outpatient. Medscape. 2021.

39. Final Opinion on Biological effects of UVC radiation relevant to health with particular reference to UVC lamps.

40. Einstein GP, Kowalczewska M, Tulp OL. Biophotonic and bioenergetic phototherapy for treatment of antimicrobial resistant $\mathrm{S}$ aureus infection [ARSI]. The FASEB Journal. 2012;26(1 Supplement):773-773.

41. Einstein GP, George P, Tulp OL. Integrated Sigma Dynamics Quantum Energy Therapy Presented by Mathematical Function and Matrices in Immunologic Stimulation. The FASEB Journal. 2018;31(S1) lb805-1b805.

42. Einstein GP, Echavarria L, Tulp OL. Application of Einstein Matrix Medical Treatment for HIV/HCV therapy. The FASEB Journal. 2020.

43. DiRuzzo J, Kerna NA. Coronavirus Now: A Timely and Practical Summary of the Current Epidemiological Information, Clinical Presentation, and Prevention and Treatment of 2019-nCoV (2019 Novel Coronavirus)". EC Emergency Medicine and Critical Care. 2020;4(4):25-31.

44. Pfizer Initiates Phase 1 Study of Novel Oral Antiviral Therapeutic Agent Against SARS-CoV-2. 2021.

45. Sanon V, Sainvil F, Frantz, Tulp O, et al. Haiti's Cholera Epidemic: When the Unexpected Must be Expected. Experimental Biology. 2021;35:S1

46. Pasquereau S, Nehme Z, Ahmad S, et al. Resveratrol Inhibits HCoV229E and SARS-CoV-2 Coronavirus Replication In Vitro. Viruses. $2021 ; 13(2): 354$

47. Zhang J, Garrett S, Sun J. Gastrointestinal symptoms, pathophysiology, and treatment in COVID-19. Genes Dis. 2020. 